\title{
Looking for Mobility of Hypothetical Venusian Fauna
}

\author{
Leonid V. Ksanfomality \\ Space Research Institute of the Russian Academy of Sciences, \\ 84/32Profsoyuznaya str., Moscow, 117997 Russia, Space Research Institute of the RAS \\ *Corresponding Author: ksanf@iki.rssi.ru
}

Copyright (C) 2013 Horizon Research Publishing All rights reserved.

\begin{abstract}
Existing data on flora and fauna of other planets are based only on scientific hypotheses. Scanning cameras of the VENERA-9 landers were intended to produce a general notion about the planet's surface and did not anticipate looking for possible inhabitants of Venus. The panorama of the planet's solid surface was transmitted historically for the first time from the surface of Venus by the VENERA-9 lander in 1975 and repeated by the VENERA-13, -14 in 1982. Data on some results of the analysis of the content of re-processed panorama of the VENERA-9 and VENERA-13, -14 landers are presented. The low noise of the VENERA-9 data allowed allocating a relatively large object of an unusual regular structure. Its complex shape having terramorphic features and its structure suggest that the object may be a real habitant of the planet. It is not excluded that another similar object observed was damaged during the VENERA-9 landing. From the evidence of their very slow movement and position of some other similar objects it is concluded that the physical action of the Venusian fauna may be much slower than that of the Earth' fauna. Another question arises is what liquid stuffs could be used by life in the conditions of the high temperature oxygenless atmosphere of the waterless planet.
\end{abstract}

Keywords Extraterrestrial Life, Hypothetic Venus Fauna, Terramorphism, VENERA Missions, Fauna Mobility Rate

\section{Introduction}

All existing suggestions on flora and fauna of other planets are based on scientific hypotheses only (e.g., Jones, 2004). However, in the scientific literature of recent years there have been reports of hypothetical signs of life on the planet Venus, based on unique re-processed images of the surface of the planet Venus derived directly from the landers of the 1975 and 1982 TV-experiments made by the Soviet VENERA missions. (Ksanfomality, 2012b; Ksanfomality, 2012 c). Other data of this kind does not exist. The results obtained using the orbital radar (Magellan at Venus, JGR, 97, $\# 8, \# 10,1992)$ refer to large areas of the surface and in the search for life forms are useless.

A method of the TV experiments on the surface of the planet Venus (Selivanov and Gektin, 2012; Selivanov et al., 1983) performed onboard the Soviet VENERA landers (1975, 1982) did not allow observing the entities in Earth-like dynamics (if any), insofar as each panorama was scanned (and transmitted in real time) for a sufficiently long time. This time reached $30 \mathrm{~min}$ for VENERA-9 (and $13 \mathrm{~min}$ for VENERA-13 and VENERA-14). Details of the landing site were as following. The landing point coordinates of the Venera 9 were $32^{\circ} \mathrm{N}$ and $291^{\circ} \mathrm{E}$; the altitude of this point over the level of the radius $6050 \mathrm{~km}$ was $1.9 \mathrm{~km}$. The temperature was $735 \mathrm{~K}\left(462^{\circ} \mathrm{C}\right)$, the pressure $9.2 \mathrm{MPa}$, the corresponding density of the atmosphere was $65 \mathrm{~kg} / \mathrm{m}^{3}$, and it consisted of $\mathrm{CO}_{2}(96.5 \%)$ and $\mathrm{N}_{2}(3.5 \%)$. The gravity on Venus is $0.90 \mathrm{~g}$. The local time was about 10 o'clock in the morning with a solar zenith distance of $37^{\circ}$. The illumination (by scattered solar light) amounted to $3-3.5 \mathrm{klx}$.

It should be noted that the TV experiments on the Venus surface was intended for general understanding of the physics of the surface of the planet, not to search for any living forms. The TV camera resolution was 21' of arc. As for hypothetical detection of living forms review articles have been published (Ksanfomality, 2012b; 2012c), and their content is not repeated here.

In the experiment rather specific objects were observed. In their morphology and behavior, they recalled living forms (Ksanfomality, 2012a; 2012b;, 2012c; 2013). The majority of the objects found were static. However, considering sequentially obtained panoramas as instantaneous photographs, noticeable displacements of objects (or even their escape from the observation field) are observed. Some of the objects formed even explicit traces of their displacement. These observations made it possible to study the dynamics of objects and to determine their displacement velocities. Below, the behavior of various objects which, hypothetically, relate to different living forms is discussed.

\section{Hypothetical Moving Objects}

This paper deals with a special, but a significant issue - the physical mobility of hypothetical fauna of Venus. For the study 2 objects of VENERA-9 panoramas were chosen: 
'Bear' and 'Victim'. Together with them, presented is a new object, 'Amisada', that was found in complex processing of VENERA-14 panoramas. All names of objects are conditional.

\subsection{Amisada}

Amisada is one of the most interesting objects of hypothetical Venusian fauna. It has been found at the central part of the VENERA-14 panorama. Information about amisada is published for the first time. "Amisada" is an abbreviation for ancient Babylonian title of clay tablets «Ammisaduqa» in which apparitions of Venus were recorded.

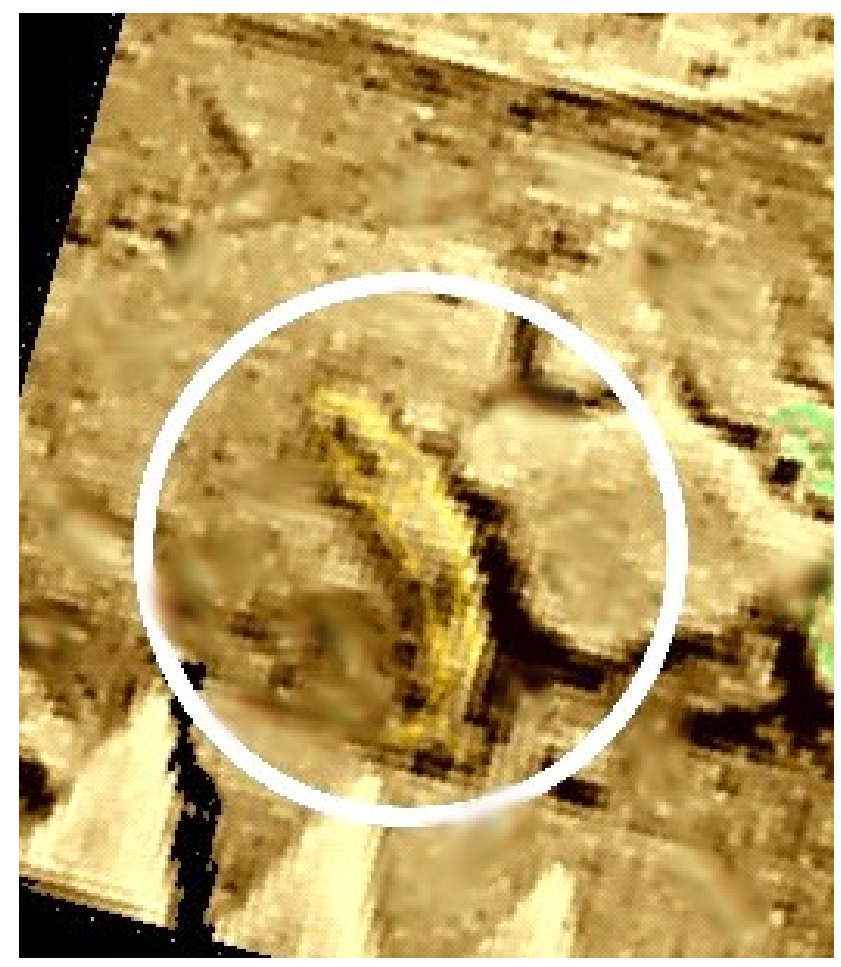

Figure.1. View of the spotty amisada. The amisada resembles a lizard climbing up on a stone. The slow displacement of the amisada's head (upper part) is detected.

Amisada is located just at the landing buffer and is marked by the white circle in Fig. 1. This object is closest to the lens of camera 1, being observed from above at an angle of about $80^{\circ}$ to the horizon. There are two other amisadas in the area but this one. The Amisada is, apparently, seen in motion. Seemingly, It is climbing up or creeping on a $5-8-\mathrm{cm}$ stone. The upper part of amisada is located on the flat surface of the stone, and the lower part envelops a ledge. Of course, it cannot be excluded that amisada does not climbe up but goes down from the stone. The other amisadas posess a "crown" at their "head" parts that is scarcely visible in Fig.1 and manifests itself by an additional processing of initial images only. Therefore, if the crown is considered as a signature of its head part, the amisada is actually climbing up onto a stone. Two small protrusions at both sides of the amisada's head could be its forelimbs. In general, it resembles a lizard climbing up to a rock. An oblong spot is seen on the head, dark spanning bands are visible at its central (at the bend) and at lower parts. The length of the amisada is about 10-12 $\mathrm{cm}$.

Animation of three subsequent frames for amisada has made it possible to detect a slight displacement of light and dark parts of its head. For 1.5 hours, the head gradually shifted first for 2 and then for 2 or 3 image pixels to opposite sides. At a distance of about $85 \mathrm{~cm}$ from the camera lens and for a resolution of $11^{\prime}$, the displacement corresponds to $1.1-1.3 \mathrm{~cm}$. In this case, the speed of the motion is significantly lower than that determined in [1-5]. It is closer to $1 \mathrm{~mm} / \mathrm{min}$, rather than $1 \mathrm{~mm} / \mathrm{s}$. This value seems to be "housekeeping action" (looking for food?) of the amisada, rather than speed of motion.

\subsection{The 'Bear-Cub'}

Terramorphism intrinsic to certain types of hypothetic Venusian fauna, apparently, relates to the deepest puzzles of searching for life in the Universe. If objects existing under quite different physical conditions exhibit, nevertheless, significant similarity of their shapes, then this phenomenon should be based on certain general natural regularities, not yet understood. In this connection, the object called 'Bear-Cub' (Fig. 2) is of a special interest. This object was found in 2012 upon additionally processing the VENERA-9 panorama (obtained back in 1975). Bear-Cub resides in the forefront of Fig. 2 in the central part of the panorama and in close proximity to the lens. In contrast to the sharp contours of the surrounding stones, the surface of the object is rather soft and even may be called fluffy. The object is located close to the photographic camera at a distance of $0.93 \mathrm{~m}$ from its optical input window and is seen almost from above at an angle of $62^{\circ}$ to the horizon. Bear-Cub seems to rest on its extremities with a certain clearance between them where the ground is seen. In this case, as is shown in Fig.2, these extremities resemble animal paws. Bear-Cub is similar in sizes to an animal like a small dog. According to the positions of shadows, which are the same for Bear-Cub and the surrounding stones, one may assume that this object is positioned vertically, with its height and length (width) being 32 to $34 \mathrm{~cm}$ and about $16 \mathrm{~cm}$, respectively. Important details can also be distinguished in Fig.2 to the left of Bear-Cub. Here, four or five long furrows 65 to $70 \mathrm{~cm}$ in length are seen. They start near the left plane stone located in the left lower part of the figure. The furrows envelop a small object, approximately $5 \mathrm{~cm}$ in size. Further, they extend under Bear-Cub and terminate immediately near its extremities. In front of Bear-Cub or, to be more precise, to the right with respect to it, there are no such tracks. Apparently, Bear-Cub is the object that has formed the furrows as a result of its motion. Thus, one is able to estimate extremely important characteristics of the motion, namely, its velocity and, possibly, even the maximum displacement velocity. 


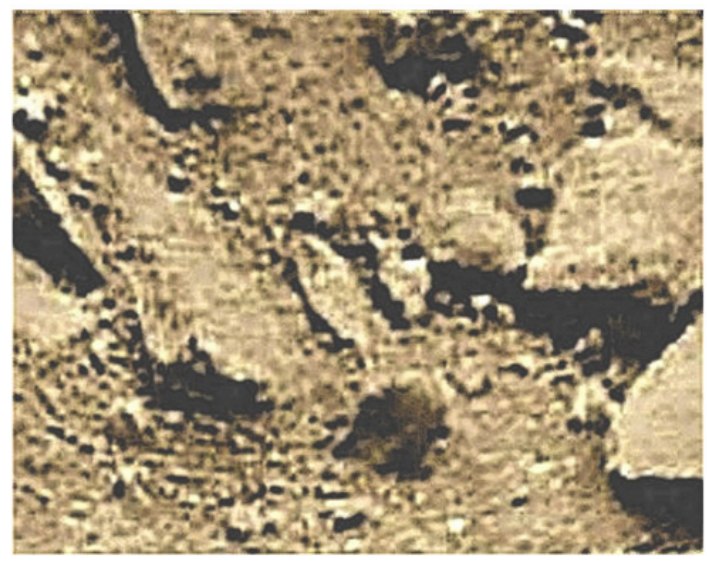

Figure 2. Panorama fragment obtained as a result of the VENERA-9 mission upon additional data processing. In the forefront, there is a small object similar to a BearCub. By its soft contours, the object is distinguished from the sharp stone edges. To the left beyond the object, over the ground, there are propagate long furrows that terminate under the BearCub. Apparently, these tracks indicate the preceding motion of the object

The velocity can be determined under the hypothetical assumption that the object attempting to escape from danger started to move at the instant of close appearance of the lander. (The lower part of Fig. 2 immediately adjoins the landing buffer of the probe.) The total time of sweeping the panorama is $30 \mathrm{~min}$, whereas the time elapsed between the start of sweeping and the scanner attaining the position of Bear-Cub was about 16 min. Under these extreme conditions, the object leaving $65-\mathrm{cm}$ tracks could develop a maximum velocity of $64 / 16=4 \mathrm{~cm} \mathrm{~min}^{-1}$, or less than $1 \mathrm{~mm} \mathrm{~s}^{-1}$. It has been already noted (Ksanfomality, 2012a; Ksanfomality, 2012b; Ksanfomality, 2012c; Ksanfomality, 2013) that the displacement velocity intrinsic to Venusian fauna seems to be rather low from the terrestrial standpoint. Is this conclusion sufficiently substantiated?

It is not excluded that the velocity of the object was significantly higher. The object could have moved much more rapidly and then have stopped at the point seen in Fig. 2. One might substantiate this assumption in the following manner. In the course of the VENERA-9 mission, two panoramas were obtained, the second one being incomplete. It covered only $124^{\circ}$, its quality was much worse than that of the first panorama and it hardly could be subjected to processing. There, Bear-Cub is not seen so clearly. However, comparison with the first panorama allows to make the following conclusion. Within the time of, approximately, 30 min that elapsed after the first scanning, the location of the object has changed negligibly and, in any case, has not exceeded several centimeters. Thus, it is quite possible that, under the critical situation, the object initially moved rapidly and then stopped prior to the sixteenth minute of scanning. Is it possible to find competing explanations for appearance of the furrows, e.g., the displacement of the object under the action of wind? In (Ksanfomality et al., 1983; Ksanfomality, 2012c), the wind velocity at the landing point of VENERA-13 was measured, which reached the value $V=0.40-0.45 \mathrm{~m} \mathrm{sec}^{-1}$. Measurements performed with other modules of the
VENERA series have given the same results (Ksanfomality, 2012c). The equivalent velocity of the Earth's wind is $8 \mathrm{~m} \mathrm{~s}^{-1}$. The possibility for Bear-Cub to be displaced under the action of wind is determined by the wind head $F=1 / 2 \rho S V^{2}$. For gas density $\rho=64 \mathrm{~kg} \mathrm{~m}^{-3}$ and the lateral surface area of Bear-Cub $S=0.05 \mathrm{~m}^{2}$, the wind pressure force attains $F=$ $0.26 \mathrm{~N}$. This force is insufficient to shift the object.

The shape of Bear-Cub, which is unusual compared to other objects found, as well as its traces and position resembling Earth's animals in the mobile state determine its high place among other candidates for Venusian fauna. One may say that alongside with other events, the VENERA-9 panoramas represent a certain demonstration area for these candidates.

\subsection{The 'Victim'}

As is seen in Fig. 3, from the point (indicated by the asterisk) on the landing buffer of the VENERA-9, a dark wide track propagates. Further, this track leaves the stone, expands and ends near a light circular entity with a sharpened left edge. There are no similar tracks on any other panoramas of the VENERA landers. The track, in itself, is very dark, similar to wet traces of running water.

However, looking for the origin of the track, one concludes that it is formed by a liquid substance of unknown origin, which cannot be water. The critical water data are $647 \mathrm{~K}$ and $22 \mathrm{MPa}$; therefore, the temperature of $735 \mathrm{~K}$ (and pressure of $9.2 \mathrm{MPa}$ ) on the Venusian surface are supercritical for water. Hence, the presence of liquid water is excluded there. Thus, this substance should be either an unknown high-temperature aqueous solution or an another unknown liquid medium.

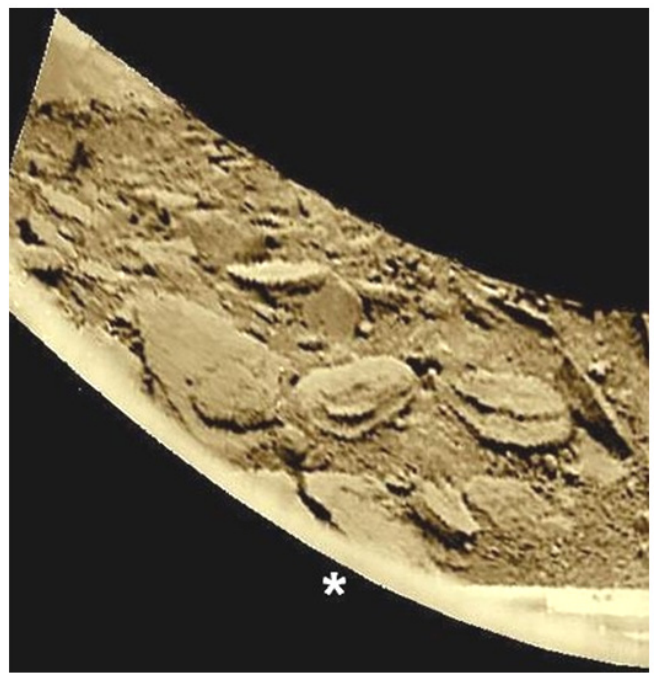

Figure. 3. Part of the VENERA-9 panorama. The dark track propagated from the point of the landing buffer (marked by asterisk) of the descent module. One may suppose that the dark track is formed by the object 'victim', wounded by the lander. The track is produced by a certain liquid substance of unknown origin (liquid water cannot exist on Venus). An object about $18 \mathrm{~cm}$ in size has managed to crawl away a distance of 26-30 $\mathrm{cm}$ in a time not longer than $6 \mathrm{~min}$ 
One may put forward the following hypothetical assumption on the origin of the track that starts immediately from the landing buffer of the descent module. If a light circular entity with a sharpened left edge does belong to Venusian fauna, it could have been damaged by the buffer, in the course of landing. In attempts to crawl away from the lander, the entity could have form the dark track of liquid substance released from its damaged tissues. For Earth' animals, this track would be called "bloody". (Thus, October 22,1975 , could be considered hypothetically to be the date of the first victim in studies of Venus by the terrestrial civilization). So the proposed nickname for the entity is a "victim".

The position of "victim" on the panorama corresponds to the sixth minute of scanning. The distance at which the object could have crawled away in this time corresponds to 26-30 cm, insofar as the scanning camera was switched on almost immediately upon landing of the module. Therefore, the elapsed scanning time (6 min) and coordinates of the "victim" location point make it possible to estimate the velocity of displacement accompanied by the formation of the track. Thus, the velocity occurs to be about $6 \mathrm{~cm} \mathrm{~min}^{-1}$, or $1 \mathrm{~mm} \mathrm{sec}$. This value is close to that found in the preceding section. The shape of "victim" (upon introducing geometric corrections) and the size scale are illustrated by the photo plan (Fig. 4), where the suffering entity rests between large stones. It is difficult to conclude whether or not the entity continues moving, insofar as the second panorama of VENERA-9 has not reach the position of "victim" to be attained.

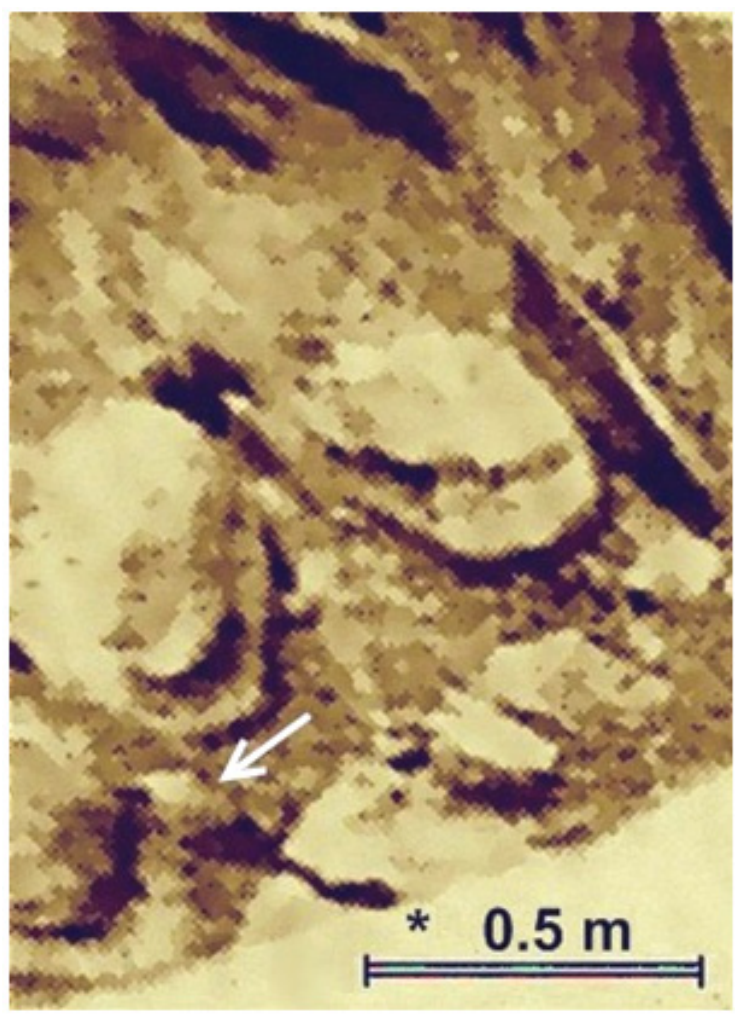

Figure.4 A fragment of a photo plan which makes it possible to measure the relative size of details and to determine their positions. The possibly wounded 'victim' is marked by arrow. An asterisk marks the beginning of the track
The dark track, in itself, indicates the fact that the objects under consideration, in the case of actual danger, are capable of moving at a velocity of not less than $1 \mathrm{~mm} \mathrm{sec}^{-1}$ (even in a damaged state). However, in other cases, displacement of objects related to the possible Venusian fauna was not observed on the panoramas of VENERA-9. Most likely, the slowness is intrinsic to Venusian fauna.

It is worth recalling that the 'Scorpion' object (Ksanfomality, 2012c) escaped from the panorama field of vision between the 93rd and 119th minutes of functioning of the lander. To this end, the object should move an estmated distance not less than $1 \mathrm{~m}$ at a velocity not less than $4 \mathrm{~cm} \mathrm{~min}^{-1}$. The conclusion also has been made for the 'Scorpion' object (Ksanfomality, 2012c) since $1.5 \mathrm{~h}$ was required for the simple operation recovering itself. One should note here that the energy resources of Earth's fauna are much greater than those of the hypothetic Venusian fauna. The reason consists in the favorable natural conditions on the Earth's surface: the abundance of flora suitable for food and the presence of the oxidation atmosphere.

As for slowness of Venusian fauna there are at least two possible explanation. First, it could be associated directly with its limited energy resources. However, there might be a different, no less plausible reason behind that, inasmuch as water, an active medium in the Earth organisms, does not exist on Venus in its liquid form and hence there should be something else instead. Unlike water, the active medium that is the basis of the Venus fauna, can feature slow plasticity. Just as an example is resin, possessing such property. This medium requires substantial time for any change of its shape.

\section{Discussion}

The only existing data of actual close-in observations of Venus' surface are the results of a series of missions of the soviet VENERA-9, 10 and VENERA -13, 14 landers, which operated 38 and 31 years ago. Since the efficiency of the VENERA landers maintained for a rather long time they produced a number of primary television panoramas during the lander's work. The images obtained admit further significant improvement of sharpness and clarification of fine details. To this end, four methods were employed: application of an unsharp-mask and the functional- sharpness methods; use of the correlative-stacking operation; and an improvement of the sharpness by consistent application of the "blur-sharpening" operation of standard WORD and WINDOWS software. All these approaches are known sufficiently well and a significant number of relevant codes are proposed for each of them. Thus, one can try to detect in them: (a) any differences in successive images (appearance or disappearance of parts of the image or change of their shape), and understand what these changes are related to (e.g., wind), or whether they are related to hypothetical habitability of a planet. Another sign (b) of the wanted object is their morphological peculiarities which distinguishes them from the ordinary surface details. 


\section{Conclusion}

The analysis of newly processed panoramas of the Venusian surface, which were returned in the course of the VENERA-9 mission of 1975 and VENERA-14 in 1982 was fulfilled. The rate of mobility of hypothetical Venusian fauna has been studied. Three hypothetic new entities, 'Amisada', 'Bear-Cub' and 'Victim' of noticeable size (about 15-20 cm) were detected in the VENERA-9 and VENERA-14 low-noise panoramas. It is difficult to explain the appearance of these objects by random reasons (by noises). No fast movements of the entities 'Amisada', 'Bear-Cub' and 'Victim' exceeding $1 \mathrm{~mm} \mathrm{~s}-1$ were found. However, for these short scanning time and long intervals between subsequent panoramas the results cannot be fully representative. Nevertheless, in these almost noise-free panoramas signs of displacement of the entities are observed. Arguments are presented testifying to the fact that the 'Bear-Cub' seem to possess a complicated regular structure. The scanning cameras of the VENERA landers were intended to produce a general notion about the planet's surface and did not anticipate finding any possible inhabitants of Venus. New investigations need for better resolution. The special mission, if it ever takes place, should be significantly more complex.

\section{REFERENCES}

[1] Jones, B.W. 2004., Life in the Solar system and beyond, Springer, Chichester.

[2] Ksanfomality, L.V., 2013. An object of assumed venusian flora. DAN Phys., 450: 39-42.

[3] Ksanfomality, L.V., 2012a. Hesperos: Unusual objects on the surface of the planet venus according to data of missions of 1975-1982. DAN Phys., 447: 28-32.

[4] Ksanfomality, L.V., 2012b. Possible detection of life on the planet Venus. DAN Phys., 446: 42-47.

[5] Ksanfomality, L.V., 2012c. Venus as a natural laboratory for search of life in high temperature conditions: Events on the Planet on March 1, 1982. Solar Syst. Res., 46: 44-57.

[6] Ksanfomality, L.V., N.V. Goroshkova and V.K. Khondirev, 1983. WIND velocity at the venus surface obtained by means of acoustic measurements. kosmich. Isslled, 2: 218-224.

[7] Selivanov, A.S., Y.M. Gektin and M.A. Gerasimov, 1983. Continuation of the tv study of the surface of venus by means of landers. Kosmich. Issled., 21: 176-182.

[8] Selivanov, A.S., Y.M. Gektin, 2012. A comment on the article by L. V. Ksanfomality "Results of the new processing of images obtained from the surface of Venus in the TV experiment onboard the VENERA-9 lander (1975)". Solar System Research, 46: 385. 